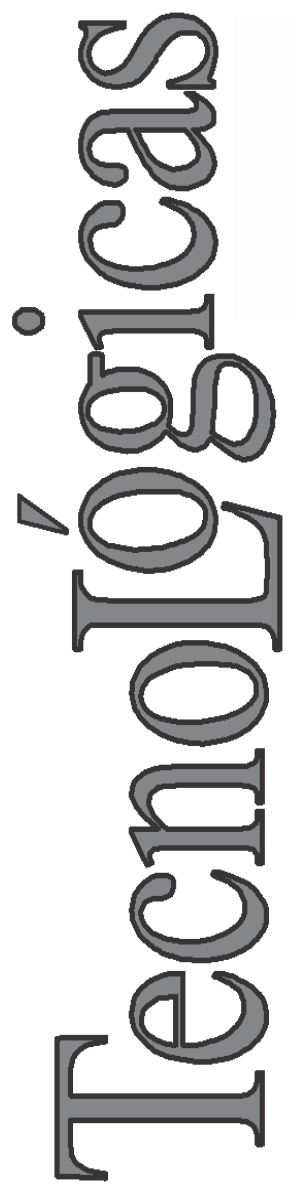

\title{
Desarrollo de un Sistema Emulador de Arreglos Fotovoltaicos en Tiempo Real Considerando Variaciones en las Condiciones Climáticas
}

\section{Development of a Photovoltaic Array Emulator System in Real Time Considering Climatic Conditions Variations}

\author{
Camilo E. Ardila-Franco ${ }^{1}$ \\ John A. Soto-Gómez ${ }^{2}$ \\ Eliana I. Arango-Zuluaga ${ }^{3}$ \\ Carlos A. Ramos-Paja 4 \\ Sergio I. Serna-Garcés ${ }^{5}$
}

1 Facultad de Minas, Departamento de Energía Eléctrica y Automática, Universidad Nacional de Colombia Sede Medellín, Medellín-Colombia ceardilaf@unal.edu.co

2 Facultad de Minas, Departamento de Energía Eléctrica y Automática, Universidad Nacional de

Colombia Sede Medellín, Medellín-Colombia jasotogo@unal.edu.co

3 Facultad de Minas, Departamento de Energía Eléctrica y Automática, Universidad Nacional de Colombia Sede Medellín, Medellín-Colombia eiarangoz@unal.edu.co

4 Facultad de Minas, Departamento de Energía Eléctrica y Automática, Universidad Nacional de

Colombia Sede Medellín, Medellín-Colombia caramosp@unal.edu.co

5 Departamento de Electrónica y Telecomunicaciones, Instituto Tecnológico Metropolitano, Medellín-Colombia sergioserna@itm.edu.co 


\title{
Resumen
}

El objetivo de este trabajo es exponer el desarrollo de un emulador que posee la capacidad de replicar, en tiempo real, el comportamiento de arreglos de paneles fotovoltaicos (PV), en los cuales cada uno de los paneles que lo conforma puede operar bajo distintas condiciones de irradiación y temperatura. El emulador está constituido por una tarjeta de adquisición de datos, una fuente programable y un computador; y está basado en el modelo del diodo de bypass, que ofrece una mejor aproximación ante condiciones reales de operación. Utiliza la función Lambert $\mathrm{W}$ en una ecuación simplificada, que disminuye el tiempo de cálculo de la solución. $\mathrm{Al}$ calcular la solución, genera una tabla de datos de los valores de corriente como una función del voltaje en terminales, temperatura e irradiación. La emulación en tiempo real se realiza por medio de un algoritmo de búsqueda, en la tabla de soluciones generada, del valor más cercano al voltaje impuesto en las terminales.

\section{Palabras clave}

Emulador de panel fotovoltaico; tiempo real; modelo del diodo de bypass; función Lambert W; fuente programable.

\begin{abstract}
This paper presents the development of an emulator that has the ability to replicate, in real time, the behavior of photovoltaic panels (PV) arrays considering different conditions of irradiation and temperature for each one. The emulator consists of a data acquisition card, a programmable source and a computer. It is based on the bypass diode model that provides a better approximation to real operating conditions. The solution is computed by a simplified equation that uses the Lambert $\mathrm{W}$ function, which reduces the computation time. After that, it generates a solution table of values of current as a function of voltage on terminals, temperature and irradiation. Real-time emulation is performed by means of a search algorithm in the solutions table of the closest value to the voltage imposed on the terminals.
\end{abstract}

\section{Keywords}

Photovoltaic panel emulator; real time; bypass diode model; Lambert W function; programmable source. 


\section{INTRODUCCIÓN}

El rápido avance en el estudio de la generación de energías alternativas haciendo uso de paneles fotovoltaicos (PV) trae consigo la necesidad de desarrollar herramientas que permitan agilizar y replicar sus características de operación (Dolan et al., 2010; Di Piazza \& Vitale, 2010; Martín-Segura et al., 2007). Con dichas herramientas se busca suprimir la dependencia de condiciones climáticas, como la temperatura e irradiación solar, ya que éstas son factores determinantes en la cantidad de energía producida por el PV, lo cual modificaría la comparación de, por ejemplo, estrategias de búsqueda del punto de máxima potencia y control.

En la búsqueda de este objetivo se han propuesto diferentes emuladores de tipo analógico y simuladores de arreglos de PV con los cuales es posible obtener información acerca del modo de operación estático, es decir a temperatura e irradiación constante, de dichos arreglos (Schofield, 2011). Sin embargo, uno de los principales objetivos de este campo de estudio en la actualidad, se enfoca en obtener la mayor cantidad de energía posible ante condiciones dinámicas de irradiación y temperatura (Petrone \& Ramos-Paja, 2011; Petrone et al., 2007)., para lo cual es necesario contar con un emulador que logre replicar las condiciones de operación de un arreglo de PV en condiciones variables de temperatura e irradiación y que además considere que no todos los elementos del arreglo están sometidos a las mismas condiciones.

El objetivo de este trabajo es exponer el desarrollo de un emulador que posee la capacidad de replicar la operación de un arreglo de PV en condiciones variables de temperatura e irradiación y que tiene en cuenta condiciones diferentes para cada panel del arreglo fotovoltaico. En la sección 2 se presenta el modelo con el cual se desarrollará el emulador y las ecuaciones para arreglos de PV. La sección 3 expone el desarrollo del emulador de arreglos de PV. En la sección 4 se presentan los resultados experimentales y ventajas de su implementación y finalmente en la sección 5 se describen las conclusiones del trabajo realizado. 


\section{MODELO DEL DIODO DE BYPASS Y ARREGLOS DE PANELES FOTOVOLTAICOS PV}

En la Fig. 1 se presenta uno de los modelos de la literatura. Este modelo implementa un diodo de bypass $(D b)$ con el fin de proteger los PV, cuando se conectan en serie, de las corrientes de otros PV que podrían llegar a ser mayores y causar daños. Las ecuaciones que describen el modelo y que han sido extraídas de Petrone et al. (2007), se presentan en (1)-(5).

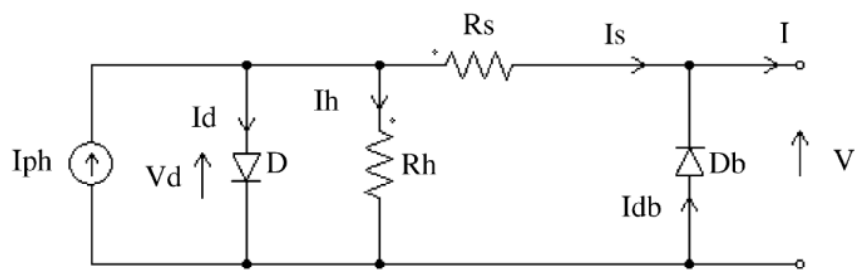

Fig. 1. Modelo circuital de PV

$$
\begin{aligned}
& I_{d}=I_{s a t, d}\left(e^{\frac{-V}{V_{t, d}}}-1\right) \\
& I_{d b}=I_{s a t, d b}\left(e^{\frac{-V}{V_{t, d b}}}-1\right) \\
& I=I_{d b}+I_{p h}-I_{d}-I_{h} \\
& V_{d}=V+R_{s} I_{s}=V+R_{s}\left(I-I_{d b}\right) \\
& I_{h}=\frac{V_{d}}{R_{h}}=\frac{V+R_{s}\left(I-I_{d b}\right)}{R_{h}}
\end{aligned}
$$

Donde $\mathrm{I}_{\mathrm{ph}}$, es la corriente producida por el PV, la cual depende del nivel de irradiación y la temperatura del arreglo. $V_{t, d}, V_{t, d b}, I_{s a t, d}$ y $\mathrm{I}_{\mathrm{sat}, \mathrm{db}}$ son los voltajes térmicos y corrientes de saturación del diodo $\mathrm{D}$ y $\mathrm{Db}$ respectivamte, los cuales dependen únicamente de la temperatura. Posteriormente, la corriente $I$ a la salida del PV, se puede expresar como una función no lineal del voltaje $V$ en las terminales del panel, haciendo uso de la función LambertW como se 
muestra en (6). (NOTA: La función LambertW $(\sigma)$ se define como la inversa de la función $\left.f(\sigma)=\sigma e^{\sigma}\right)$.

$I=\frac{\left[R_{h}\left(I_{p h}+I_{s a t, d}\right)-V\right]}{R_{h}+R_{s}}+I_{s a t, d b}\left(e^{\frac{-V}{V_{t, d b}}}-1\right)-\frac{V_{t, d}}{R_{s}} \operatorname{LambertW}(\theta)$

$\theta=\frac{\left(R_{h} / / R_{s}\right) I_{s a t, d} e^{R_{h} R_{s}\left(I_{p h}+I_{s a t, d}\right)+R_{h} V / V_{t, d}\left(R_{h}+R_{s}\right)}}{V_{t, d}}$

A pesar de la precisión del modelo de la Fig. 1, el alto costo computacional que requiere para obtener la solución de las ecuaciones que lo representan, debido a la implementación de la función LambertW, hace que en ocasiones no sea posible su solución en arreglos formados por varios paneles conectados en serie. Las ecuaciones que describen este tipo de arreglos se presentarán más adelante.

Sin embargo, es posible hacer simplificaciones sobre dicho modelo, de tal forma que la solución encontrada no requiera un costo computacional tan alto como el del modelo original. Una de las simplificaciones usadas es despreciar el valor de $\mathrm{R}_{\mathrm{s}}$ en el modelo de la Fig. 1. Esta aproximación no introduce un gran error en los cálculos numéricos y disminuye notablemente el tiempo de cálculo, debido a que la resistencia $R_{s}$ toma valores cercanos a $0.006 \Omega$ (Petrone et al., 2007). Con esta simplificación, la corriente I a la salida del panel está dada por (8)

$I=I_{s a t, d b}\left(e^{\frac{-V}{V_{t, d b}}}-1\right)+I_{p h}-I_{s a t, d}\left(e^{\frac{-V}{V_{t, d}}}-1\right)-\frac{V}{R_{h}}$

Ahora bien, regularmente, los PV se encuentran conectados en arreglos en serie (string), paralelo o mixtos; en la Fig. 2 se muestra un arreglo en serie. La forma de conexión del arreglo de paneles y las condiciones de irradiación y temperatura de cada panel, determinan el voltaje $V$ y la corriente $I$ que serán entregadas en las terminales de todo el arreglo. 


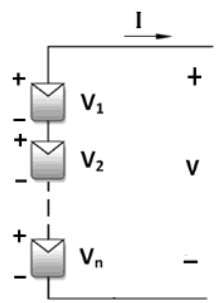

Fig. 2. $n$ paneles conectados en serie

Aplicando la ley de voltajes de Kirchoff en el arreglo de n paneles en serie de la Fig. 2, se tiene que:

$\mathrm{V}_{1}+\mathrm{V}_{2}+\mathrm{V}_{3}+\cdots+\mathrm{V}_{\mathrm{n}}=0$

Teniendo en cuenta que la corriente que pasa por todos los paneles es la misma se tiene que:

$$
\left\{\begin{array}{c}
\mathrm{I}_{1}\left(\mathrm{~V}_{1}\right)-\mathrm{I}_{2}\left(\mathrm{~V}_{2}\right)=0 \\
\mathrm{I}_{1}\left(\mathrm{~V}_{1}\right)-\mathrm{I}_{3}\left(\mathrm{~V}_{3}\right)=0 \\
\ldots \ldots \ldots \ldots \ldots \ldots \ldots \ldots \ldots \ldots \ldots \ldots \ldots \\
\mathrm{I}_{1}\left(\mathrm{~V}_{1}\right)-\mathrm{I}_{\mathrm{n}-1}\left(\mathrm{~V}_{\mathrm{n}-1}\right)=0 \\
\mathrm{I}_{1}\left(\mathrm{~V}_{1}\right)-\mathrm{I}_{\mathrm{n}}\left(\mathrm{V}_{\mathrm{n}}\right)=0
\end{array}\right\}
$$

Donde $\mathrm{I}_{\mathrm{j}}, \mathrm{V}_{\mathrm{j}}$ son la corriente $\mathrm{j}$ y el voltaje $\mathrm{j}$ del panel $\mathrm{j}$ del arreglo en serie, respectivamente, para $j=1,2,3, \ldots \ldots, n$. La relación entre $I_{j}$ y $V_{j}$ esta dada por (6), (8) ó cualquier otra ecuación resultante del modelamiento del panel. Las ecuaciones (9) y (10), conforman un sistema de $\mathrm{n}$ ecuaciones linealmente independientes cuyas $\mathrm{n}$ incógnitas se encuentran al solucionar el sistema. De esta manera queda descrito totalmente el comportamiento del arreglo en serie de PV.

En el caso de que el arreglo de PV estuviese conectado en paralelo, se obtiene la solución de las ecuaciones de modelamiento para cada panel por separado y posteriormente se suman las corrientes producidas por cada uno para obtener la corriente total. Si la conexión es mixta, inicialmente se calculan las corrientes de los arreglos en serie y luego se suman para obtener la corriente total 


\section{EMULADOR DE ARREGLOS DE PANELES FOTOVOLTAICOS PV}

El emulador propuesto en este artículo es capaz de replicar el comportamiento de arreglos de PV, en los cuales cada uno de los paneles que lo conforma puede operar bajo distintas condiciones de irradiación y temperatura. Para lograr esto se hace uso de una tarjeta de adquisición de datos, una fuente programable y un computador.

El objetivo de la tarjeta es controlar, mediante una salida analógica, el valor de la corriente que entrega la fuente programable, la cual funciona como fuente de corriente controlada por voltaje. El valor de corriente $\mathrm{I}_{\text {ref }}$ que envíe la tarjeta a la fuente, dependerá del voltaje a la salida del arreglo, el modelo de panel utilizado y el algoritmo implementado para calcular la corriente del arreglo como una función del voltaje, la temperatura y la irradiación. Además con una entrada analógica de la tarjeta de adquisición, se tomará la medida del voltaje en las terminales del arreglo, y de esta medida dependerá el valor de corriente que será impuesto en la fuente programable como se mencionó anteriormente. En la Fig. 3 se presenta el diagrama de bloques del emulador propuesto.
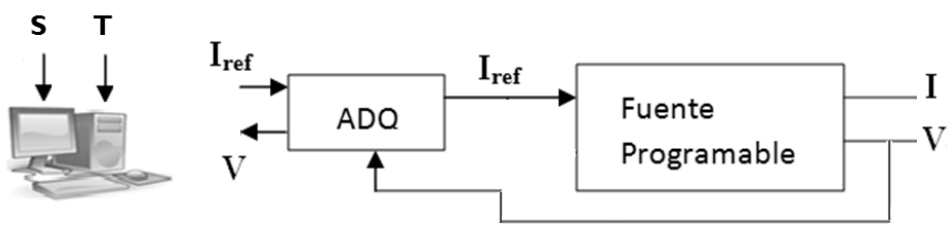

Fig. 3. Diagrama de bloques del emulador

Debido a que la operación anteriormente mencionada se debe realizar en tiempo real, ya que el lazo de control está realimentado, es necesario que el algoritmo implementado en el computador sea lo suficientemente rápido para lograr este fin. Sin embargo la solución del sistema conformado por (9) y (10), se calcula tantas veces como número de arreglos en serie tenga el arreglo total. Además se realiza el cálculo del arreglo completo para cada par temperatura - irradiación dado, los cuales pueden variar con el tiempo según el perfil de condiciones climáticas introducido por el 
usuario. Dicho perfil especifica las condiciones de temperatura e irradiación de cada panel del arreglo para cada instante de tiempo.

Finalmente se encuentra la solución de un modelo discreto, la resolución de la respuesta encontrada, dependerá de la cantidad de puntos de voltaje para los cuales se quiera conocer la corriente ante todas las condiciones mencionadas anteriormente. Por supuesto a medida que aumente la cantidad de puntos de voltaje, aumentará la precisión del cálculo realizado respecto al modelo continuo, al igual que el consumo de recursos computacionales. En la Tabla 1, se presentan los tiempos de cálculo de la solución para un arreglo de tres paneles en serie, tomando en cuenta cantidades diferentes de puntos de voltaje y diez y nueve pares de datos de temperatura - irradiación. El modelo de PV utilizado para encontrar la solución es el presentado en (8).

Tabla 1. Tiempos de cálculo de solución según la resolución en el eje de voltaje.

\begin{tabular}{cc}
\multicolumn{2}{c}{ Fuente: Autores. } \\
\hline Número de puntos & Tiempo de cálculo (s) \\
\hline 100 & 76.15 \\
200 & 145.18 \\
300 & 197.52 \\
400 & 270.85 \\
\hline
\end{tabular}

$\mathrm{Al}$ observar los tiempos de cálculo presentados en la tabla I, se concluye que son demasiado grandes para lograr una emulación en tiempo real que a su vez sea precisa. Para evitar este inconveniente el emulador opera de tal forma que, primero se calculan los valores de corriente para todas las condiciones de irradiación y temperatura dadas, y para los valores de voltaje escogidos por el usuario, según la cantidad de puntos en las que se haya dividido el eje de voltaje. Los resultados de este cálculo se puede observar en las Fig. 4 y 5, que muestran las gráficas de corriente contra voltaje y de potencia contra voltaje, para diferentes condiciones de irradiación y temperatura en cada panel del arreglo (las cuales son variantes en el tiempo). Los datos tomados para el cálculo y la simulación son los mismos de la Tabla 1. 


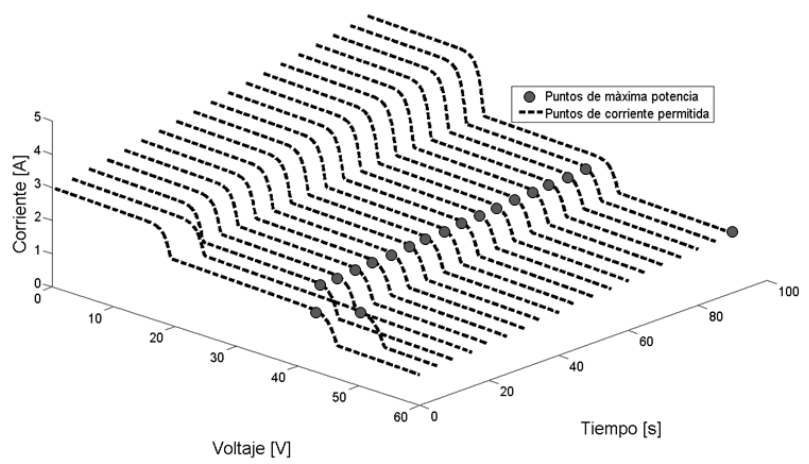

Fig. 4. Curvas de corriente contra voltaje calculadas para condiciones de temperatura e irradiación variantes en el tiempo

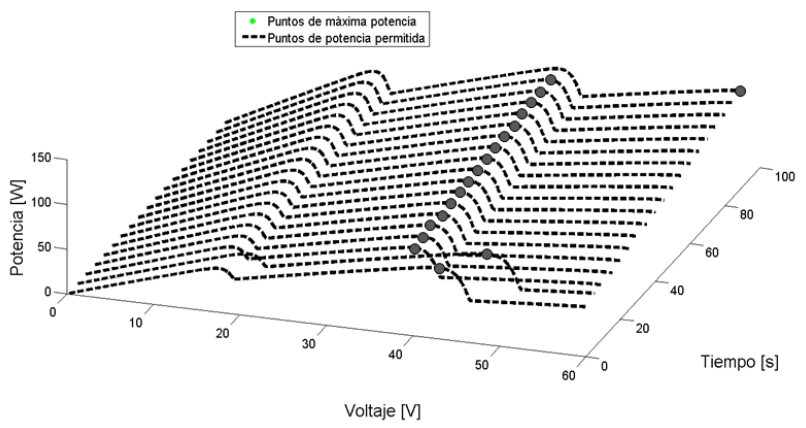

Fig. 5. Curvas de potencia contra voltaje calculadas para condiciones de temperatura e irradiación variantes en el tiempo

Una vez obtenidos los valores para la corriente como una función del voltaje, la temperatura y la irradiación; se genera una tabla de datos de voltaje en las terminales del arreglo y su valor de corriente correspondiente. De esta manera, el único algoritmo que se debe ejecutar en tiempo real para lograr la emulación, es realizar la búsqueda en la tabla de datos del valor más cercano al voltaje impuesto en las terminales para obtener el valor correspondiente de la corriente $\mathrm{I}_{\text {ref }}$ que será enviada por la tarjeta a la fuente programable. Por su sencillez, este algoritmo de búsqueda tiene un tiempo bajo de ejecución, lo que facilita la emulación en tiempo real. En las Fig. 6 y 7, se puede observar el resultado de la emulación de los datos mostrados en las Fig. 4 y 5 respectivamente. 


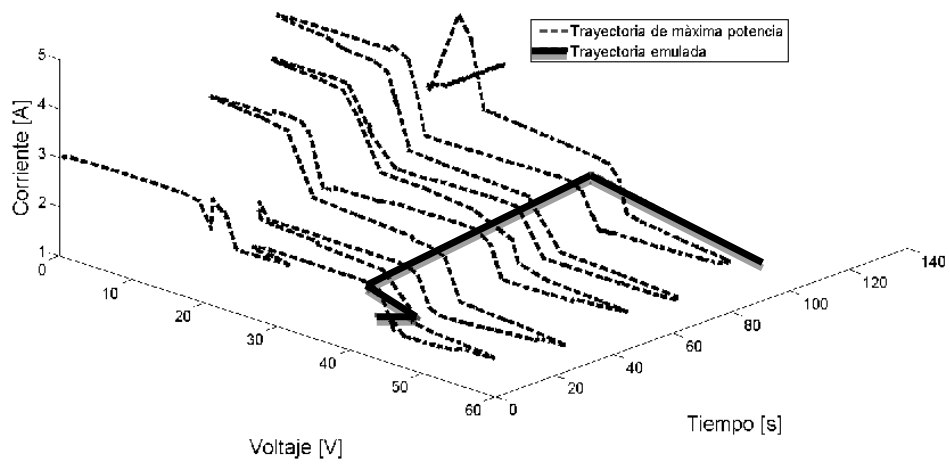

Fig. 6. Curva de corriente contra voltaje emulada para diferentes condiciones de temperatura e irradiación variantes en el tiempo

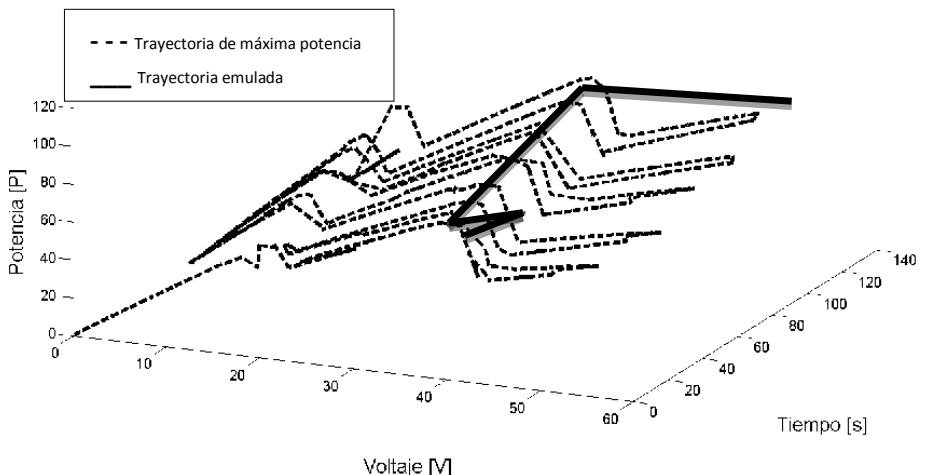

Fig. 7. Curva de potencia contra voltaje emulada para diferentes condiciones de temperatura e irradiación variantes en el tiempo

\section{RESULTADOS EXPERIMENTALES}

Para la realización de este experimento se utilizó una fuente programable "BK PRECISION XLN6024", una carga programable "BK PPRECISION 8514", una tarjeta de adquisición de datos "NI 3663 " con un bloque de conectores "NI SCB-68" y un computador con Matlab. 


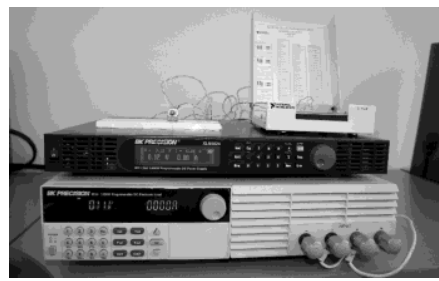

Fig. 8. Foto real del emulador

La prueba experimental consiste en conectar una carga resistiva a la salida del emulador, de esta manera, los valores de la corriente y voltaje a la salida para la carga resistiva, serán los correspondientes a la intersección entre la gráfica que describe el comportamiento del emulador $(I=f(v, t))$, y una recta de pendiente $\frac{1}{R}$ con un punto de corte en el origen de la gráfica $I=f(V)$, donde $R$ es el valor de la carga a la salida en $\Omega$.

En la Fig. 9, se ilustran los resultados obtenidos para un par de condiciones de temperatura e irradiación dados, y para 21 valores diferentes de resistencias que varían desde 0 a $65.22 \Omega$ (La inclinación de la recta aumenta a medida que disminuye la resistencia).

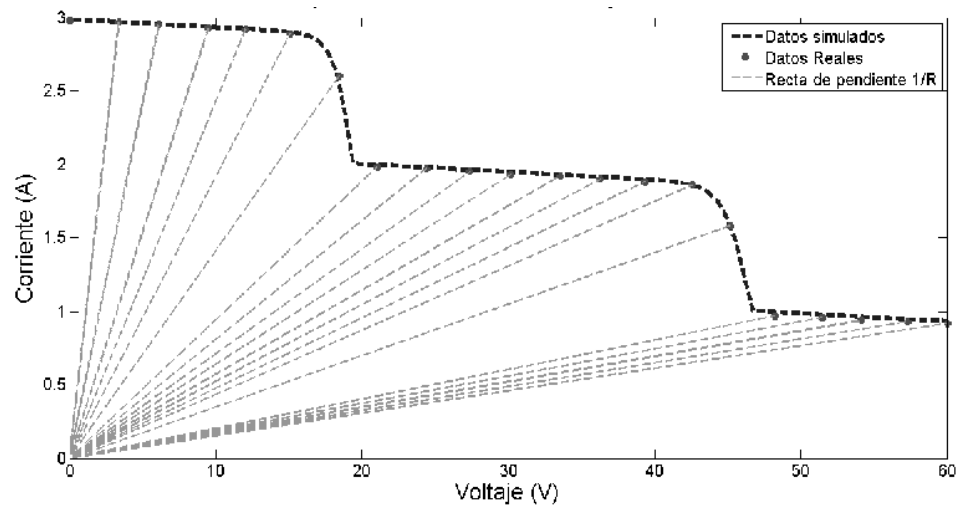

Fig. 9. Comparación de datos simulados y datos reales para diferentes cargas resistivas 
Para comparar la similitud de los datos emulados (reales), contra los datos calculados (teóricos), se utilizó el error medio cuadrático entre los datos de corriente para cada una de las cargas, y la corriente correspondiente al punto de la gráfica $\mathrm{I}=\mathrm{f}(\mathrm{t}, \mathrm{v})$ con voltaje más cercano al voltaje real. Se obtuvo un error cuadrático medio de 0.2828, que demuestra la aproximación lograda con el emulador en funcionamiento.

\section{CONCLUSIONES}

Es posible emular en tiempo real el comportamiento de arreglos de paneles fotovoltaicos, independientemente del modelo utilizado, la cantidad de datos o la variación en las condiciones, calculando previamente las soluciones y realizando la emulación en una etapa posterior.

A pesar de que el cálculo de la curva voltiampérica del panel fotovoltaico es un problema continuo, es necesaria su discretización de tal modo que se pueda encontrar una solución para un número de puntos de voltaje finito, de este número de puntos dependerá el error de emulación y el tiempo de cálculo de la solución. Se obtuvo un buen funcionamiento del emulador desarrollado, el cual fue comprobado por medio de resultados experimentales.

\section{AGRADECIMIENTOS}

Este trabajo ha sido desarrollado en la línea de investigación en Modelado y control de fuentes de potencia eléctrica del Grupo de Automática de la Universidad Nacional GAUNAL, bajo los proyectos "MPPT Vectorial" y "Smart-Alen", en colaboración con el grupo de Automática y Electrónica del Instituto Tecnológico Metropolitano ITM.

\section{REFERENCIAS}

Dolan, D., Durago, J., \& Crowfoot, J. (2010, September). Simulation of a photovoltaic emulator. In North American Power Symposium (NAPS), 2010 (pp. 1-7). IEEE. 
Di Piazza, M. C., \& Vitale, G. (2010). Photovoltaic field emulation including dynamic and partial shadow conditions. Applied Energy, 87(3), 814-823.

Martín-Segura, G., López-Mestre, J., Teixidó-Casas, M., \& SudriàAndreu, A. (2007, October). Development of a photovoltaic array emulator system based on a full-bridge structure. In Electrical Power Quality and Utilisation, 2007. EPQU 2007. 9th International Conference on (pp. 1-6). IEEE.

Petrone, G., \& Ramos-Paja, C. A. (2011). Modeling of photovoltaic fields in mismatched conditions for energy yield evaluations. Electric Power Systems Research, 81(4), 1003-1013.

Petrone, G., Spagnuolo, G., \& Vitelli, M. (2007). Analytical model of mismatched photovoltaic fields by means of Lambert W-function. Solar Energy Materials and Solar Cells, 91(18), 1652-1657.

Schofield, D. M. K., Foster, M. P., \& Stone, D. A. (2011). Low-cost solar emulator for evaluation of maximum power point tracking methods. Electronics Letters, 47(3), 208-209. 